\title{
LA RECUPERACIÓN DE LAS RELACIONES TRANSFRONTERIZAS ENTRE LOS VALLES DE ANSÓ Y RONCAL COMO MODELO DE COLABORACIÓN PARA EL FUTURO
}

\author{
Antonio Jesús Gorría Ipas \\ Dpto. de Geografía - Facultad de Filosofía y Letras, Universidad de Zaragoza \\ ajgorria@unizar.es
}

\begin{abstract}
Resumen: El espacio pirenaico se caracteriza por su gran diversidad tanto física como socioeconómica y cultural. Durante siglos, el aprovechamiento de sus recursos naturales (pastos, agua, bosques... ) ha dado lugar a conflictos entre las distintas comunidades, perteneciesen o no a la misma vertiente de aguas, región o país. Fue precisamente la regulación de dichos aprovechamientos lo que generó lazos de unión entre los valles tratando precisamente de superar los conflictos, de tal forma que se generaban interrelaciones, funcionando a modo de espacios integrados. Mas recientemente, diversos procesos políticos y sobre todo los cambios en los modos de vida y valoración de los recursos tradicionales favorecieron el distanciamiento, cobrando fuerza el concepto de frontera, más que física, social, cultural y económica. En los últimos años, y en buena medida de la mano de políticas comunitarias (de la UE) se crean nuevos marcos de cooperación, superando ese "mundo de espaldas", cobrando fuerza de nuevo proyectos comunes en el aprovechamiento y gestión de los recursos, avanzando de nuevo hacia un espacio más integrado.
\end{abstract}

Palabras clave: Cooperación transfronteriza, desencuentros, Pirineos, cambio de usos.

\begin{abstract}
Pyrenean area is characterized by its great diversity, both physically and socio-economic and cultural. For centuries, the exploitation of natural resources (pasture, water, forest... ) led to conflicts between communities, regardless of water slope, region or country. Just the regulation of these exploitations led to links between the valleys, trying to overcome conflicts. Thereby interrelationships were created that
\end{abstract}

Recibido: 2-11-10. Aceptado: 2-2-12. 
operated as integrated spaces. Different political processes and especially lifestyles changes and traditional resource assessment favoured the distance, gaining strength the border concept over a physical social, cultural and economic concept. In recent years, due to EU policies, new frameworks for cooperation were created and eventually the "world behind" concept was overcome and again gaining strength common projects in the development and management of resources, moving forward to and more integrated space.

Keywords: cross-border cooperation, discount, Pyrenees, uses change.

\section{Introducción}

A lo largo de la historia los valles pirenaicos han funcionado como un espacio integrado dando lugar a abundantes interrelaciones entre los habitantes de ambas vertientes, tanto norte-sur como este-oeste. El espacio que nos ocupa reúne como singularidades no solamente el pertenecer a dos Estados diferentes sino a tres regiones (o Comunidades Autónomas en el caso de España) como son Aquitaine (Bearne), Navarra (Valle de Roncal) y Aragón (Valle de Ansó). La lucha por el control del espacio o mejor dicho aprovechamiento de los recursos naturales (pastos, agua, bosques...) generó conflictos que han dado lugar a una amplia bibliografía ${ }^{1}$, muy interesante para conocer las relaciones transfronterizas en el pasado (Balcells, 1976). Pero lo que se pretende con esta aportación es sintetizar algunas conclusiones de trabajo de campo $^{2}$ que trata de estudiar como se interviene desde distintas administraciones en un territorio que es homogéneo desde muchos puntos de vista, y cuales son los resultados de la aplicación de políticas diferentes, o al menos no coordinadas.

Este territorio cuenta con una rica y compleja historia común. Aquí confluyen factores físicos que de alguna forma singularizan cada valle y a la vez les hacen com-

\footnotetext{
${ }^{1}$ Bibliografía que apenas haremos referencia puesto que se refiere fundamentalmente a las relaciones del pasado y lo que nos interesa en este trabajo es presentar acciones o proyectos de futuro que en la actualidad se están impulsando, en un nuevo escenario de acercamiento. No obstante, junto a la bibliografía expuesta destacamos: Descheemaeker, 1., 1945.- Une frontière inconnue, les Pyrénées de lòcean à l'Aragon. Université de Paris; Fernandez de Casadevante. C, 1989.- La frontera hispano-francesa y las relaciones de vecindad. Donostia; Landabaso, K., 1991.- Cooperación transfronteriza y política regional comunitaria. Dirección General de Políticas Regionales, Bruselas; VV.AA, 2008, Coord, por Luís Silván.Fronteras y globalización: Europa-Latinoamérica. Prensas Universitarias de Zaragoza. Zaragoza.

${ }^{2}$ Me estoy refiriendo a un trabajo que este autor viene realizando de forma sistemática desde hace más de 8 años en el que se analiza con mayor detalle que el que nos permite este artículo los procesos de desencuentro y reencuentro que se han producido, o se están produciendo, en un espacio frontera como el Pirineo.
} 
plementarios. El clima, la altitud el relieve... han condicionado el desarrollo de diversos recursos básicos en el pasado (pastos, agua, bosques) sobre todo los relacionados con el aprovechamiento ganadero, la principal fuente de recursos en la sociedad tradicional. El aprovechamiento de estos recursos está en el origen de los anteriormente citados conflictos transfronterizos.

Nos interesa también plantear como las relaciones transfronterizas se van adaptando a la constante transformación que con el paso del tiempo experimentan los recursos y usos del territorio. Todo ello, dentro de un proceso muy dinámico, que primero fue de alejamiento entre las distintas comunidades del espacio pirenaico y, en estos momentos, de reencuentro dentro de unos marcos de ordenación de un espacio supraregional como es la UE.

\section{Un territorio integrado en el pasado}

El historiador francés Cavailles (1910) señalaba las dificultades que tenían los habitantes del Pirineo para gestionar sus recursos: pastos, agua, leñas cuando estos se encontraban en zonas limítrofes y las comunidades de ambas vertientes se consideraban con derechos para aprovechar tales recursos.

Su aprovechamiento y gestión generó muchos conflictos, algunos de ellos muy violentos, pero también dieron lugar a la firma de Tratados de Vecindad (Tratados de Paz o Passeries) que constituyen elementos clave de la cultura pirenaica. Elementos culturales que en estos momentos se pretende recuperar en un contexto de aprovechar las oportunidades que nos ofrece la historia para integrarla como un nuevo valor en alza de los recursos del Pirineo.

\subsection{El Tratado de las tres vacas. Entre los valles de Roncal (Navarra) y Baretous (Aquitaine)}

Quizá el más conocido de los conflictos y pactos surgidos en el conjunto del Pirineo fue el "Tratado de las Tres Vacas" que tuvo su origen en los aprovechamiento de unas fuentes y pastos limítrofes enfrentando a los habitantes de los valles de Roncal y Baretous. Para solucionar dicho conflicto intervinieron en un principio los Órganos Jurídicos del vizcondado de Bearne (el vizconde Gastón) y del reino de Navarra (Carlos II). Unos y otros fracasaron y al final las dos partes en conflicto llamaron a los ansotanos (Aragón) para que actuasen como jueces. Así, el 6 de octubre de 1375 "seis hombres buenos de Ansó" con su alcalde Sancho Gracia a la cabeza, dictaron sentencia en un juicio que tuvo como marco el atrio de la iglesia parroquial de San Pedro de Ansó. 
La sentencia de los ansotanos obligaba a los baretoneses a pagar a los roncaleses, en lo sucesivo y el 13 de julio de cada año, tres vacas "de dos años, de un mismo pelaje y cornaje y sin tacha de lesión alguna”. Además, bajo el control de los ansotanos se amojonaban los límites entre los puertos conflictivos y se indicaban las normas de regulación del aprovechamiento de pastos y aguas que habían sido la causa principal del conflicto.

Entre los aspectos que interesa retener destaca el hecho de que eligiesen a los ansotanos como árbitros, sin que los franceses viesen en ello posibles riegos de parcialidad por pertenecer a la misma vertiente, pues no existían en esos momentos unos conceptos de frontera, Estado o Nación en los valles pirenaicos tan excluyentes como se entendieron posteriormente. El fallo fue eficaz pues a partir de ese momento no se conocen nuevos conflictos como los ocurridos hasta entonces. Ello demuestra que los hombres de las montañas eran los mejor capacitados, por conocer las costumbres, derechos y formas de vida, para emitir unos veredictos más justos, o efectivos, que los de los Órganos Jurídicos del Vizcondado de Bearne o el reino de Navarra. Este rito se sigue practicado en la actualidad el 13 de julio si bien no tiene como objeto solucionar conflictos, no tiene sentido en estos momentos, sino planificar acciones conjuntas, proyectos de desarrollo económico, reivindicaciones comunes de infraestructuras...

\subsection{El puerto e ibón de Estanés (facería entre los valles de Ansó (Aragón) y Aspe (Bearne)}

Otro ejemplo de gestión conjunta lo tenemos en el puerto e ibón de Estanés perteneciente al término municipal de Ansó, a pesar de hallase en la vertiente norte del Pirineo. ${ }^{3}$ En este espacio, los pastores ansotanos carecen de leña y los franceses no cuentan con pastos suficientes, la solución fue un pacto mediante el cual los pastores ansotanos se abastecían de leña en el bosque francés de Aspe y estos últimos podían pastar 1 de cada 4 años en el puerto ansotano de Estanés (ver Fiaren-Guillén, 1956 y Caussimont, 1981). Recientemente, a principios del siglo XX, a este tratado se le unió el aprovechamiento hidroeléctrico del ibón por parte de los franceses.

Con relación a esta facería, también se celebraba anualmente una feria entre ambos valles para el intercambio de ganado y productos comerciales complementarios entre ambas vertientes (Gorría, 1995).

\footnotetext{
${ }^{3}$ Es uno de los pocos ejemplos en los que la frontera hispano-francesa no sigue la divisoria de aguas a pesar de los intentos que en los Tratados de Límites del siglo XIX se hizo para que coincidiesen ambos criterios.
} 


\subsection{La Junta de Puyeta, o Junta de San Miguel, regulaba conflictos y daba lugar a proyectos comunes entre los Valles de Roncal y Ansó}

Semejantes a estos Tratados de Paz, existía la costumbre de celebrar Juntas Anuales entre valles vecinos perteneciesen a la misma o distinta vertiente. Este es el caso de la Junta de San Miguel (que se celebraba por estas fechas hacia finales de septiembre). Los dos valles se reunían en la plana y ermita de Puyeta por ser lugar limítrofe, la celebración de dicha Junta se perdió cuando las necesidades de pastos dejaron de ser apremiantes y, como consecuencia, no se generaban situaciones conflictivas. $^{4}$

En estas Juntas, una vez expuestas las infracciones se pasaba a plantear acciones o proyectos de interés común. Esta tradición, o modo de actuar, se recuperó en el año 2008 con el objetivo tanto de recuperar relaciones tradiciones como, sobre todo, de planificar acciones de interés común como expondremos más adelante,

\section{El proceso de desintegración}

Diferentes procesos de desarrollo y ordenación territorial de ámbito estatal y regional determinaron que estos valles fuesen perdiendo buena parte de su capacidad de decisión pasando a convertirse en periféricos respecto a sus regiones o comunidades autónomas. Nos centraremos en dos de ellos.

\subsection{Las comunicaciones convierten al Pirineo en un espacio marginal}

El impulso de ciertas vías de comunicación favorecieron que los valles pirenaicos se volcaran hacia las cabeceras de sus respectivos llanos. Los sistemas de comunicaciones transpirenaica tradicionales que atravesaban fácilmente el Pirineo por los collados fueron perdiendo funcionalidad al no convertirse en carreteras, dando lugar a un alejamiento entre los valles de ambas vertientes, lo que se ha definido como el nacimiento de un "mundo de espaldas" (Bielza de Ory y Dalla-Rossa, 1988). En ciudades del piedemonte francés como Mauleon donde se impulsó una activa industria ${ }^{5}$ y otras cabeceras del Pirineo Central (Lannemezan, Bagneres de Bigorre, Saint-Girons...) favorecieron que los habitantes de los altos valles franceses se "volcasen" hacia esas ciudades y fueran perdiendo interés en conservar las relaciones agroganaderas tradi-

${ }^{4}$ También es necesario resaltar que conforme se afirman los Estados nacionales, y sobre todo a partir de 1860 con el Tratado de Límites franco-español, los valles pirenaicos van perdiendo "competencias" para planificar acciones transnacionales.

${ }^{5}$ La industria de alpargatas de Mauleón llegó a exportar a Argentina y atraía trabajadores de la vertiente española, algunos desde Zaragoza. 
cionales con sus vecinos españoles. Estas eran menos atractivas económicamente que las generadas en el contexto de desarrollo industrial.

\subsection{Las políticas regionales contribuyen a incrementar las diferencias entre distintos ámbitos pirenaicos}

Dentro de las políticas regionales recientes debemos considerar el apoyo que recibieron a los valles del Pirineo francés con programas que el gobierno de ese país impulsó para hacer frente a la competencia que se generaba para el sur de Francia con el ingreso de España y Portugal en la CEE. En 1980 se aprueba el Plan du Grand Sud-Ouest y una serie de Contratos Estado-Región cuyo primer resultado fue incrementar entre 1980-82 un 50\% las inversiones y subvenciones del Estado en el Pirineo. A estos primeros planes siguieron los Programas Integrados Mediterráneos (entre 1986-90), o los Programas Nacionales de Interés Comunitarios (PNIC) y Operaciones Integradas de Desarrollo (OID) que impulsaron el desarrollo de las regiones francesas del sur, favorecidas además por una política específica de macizos y áreas de montaña. Se incrementaban de esta forma las diferencias de oportunidades entre los habitantes pirenaicos de ambas vertientes.

En cuanto a la vertiente española, la configuración del Estado Autonómico dio lugar a un proceso de ordenación y desarrollo no coordinado que incrementó las diferencias de oportunidades entre regiones. Así, en Navarra se aplican programas a partir de 1986, como el DIADENA, ${ }^{6}$ que favorecieron el desarrollo de sus valles pirenaicos con inversiones en todos los sectores económicos de actividad. En los valles aragoneses del Pirineo occidental los primeros programas de desarrollo local con cierta entidad llegan mucho más tarde, no antes de la tercera convocatoria de la Iniciativa Comunitaria Leader; es decir el Leader Plus programado para 2000-2006. De lo anterior se deduce que los valles de ambas vertientes y regiones experimentan procesos de desarrollo no coordinados y con diferentes oportunidades, tanto en el tiempo como en la disponibilidad de recursos.

\section{De nuevo el reencuentro}

Se apoya en la nueva política de la UE, especialmente cuando ésta tratar de eliminar las fronteras interiores y crea Iniciativas como el INTERREG, orientadas a favorecer las relaciones entre ambas vertientes. Pero, en opinión de la población autóctona, apenas han beneficiado a los valles dado que su ámbito de acción eran las regiones y no se produjo una "discriminación positiva" para las poblaciones fronteri-

${ }^{6}$ Programa para el Desarrollo Integrado de las Áreas Desfavorecidas de Navarra. 
zas del Pirineo. No obstante, en los últimos años vemos un acercamiento entre los valles de este espacio, espontáneo, que nace de la población y las corporaciones locales.

\subsection{La recuperación de la "memoria histórica" como referente para planificar el futuro}

Además de la cada vez mayor participación en las fiestas patronales ${ }^{7}$ los programas orientados al desarrollo e incluso a la ordenación territorial se están impulsando con efectividad. Tanto la rememoración de acontecimientos históricos como el Tratado de las tres vacas y sobre todo ${ }^{8}$ el de la Junta de San Miguel favorecen el impulso de programas de fomento coordinados.

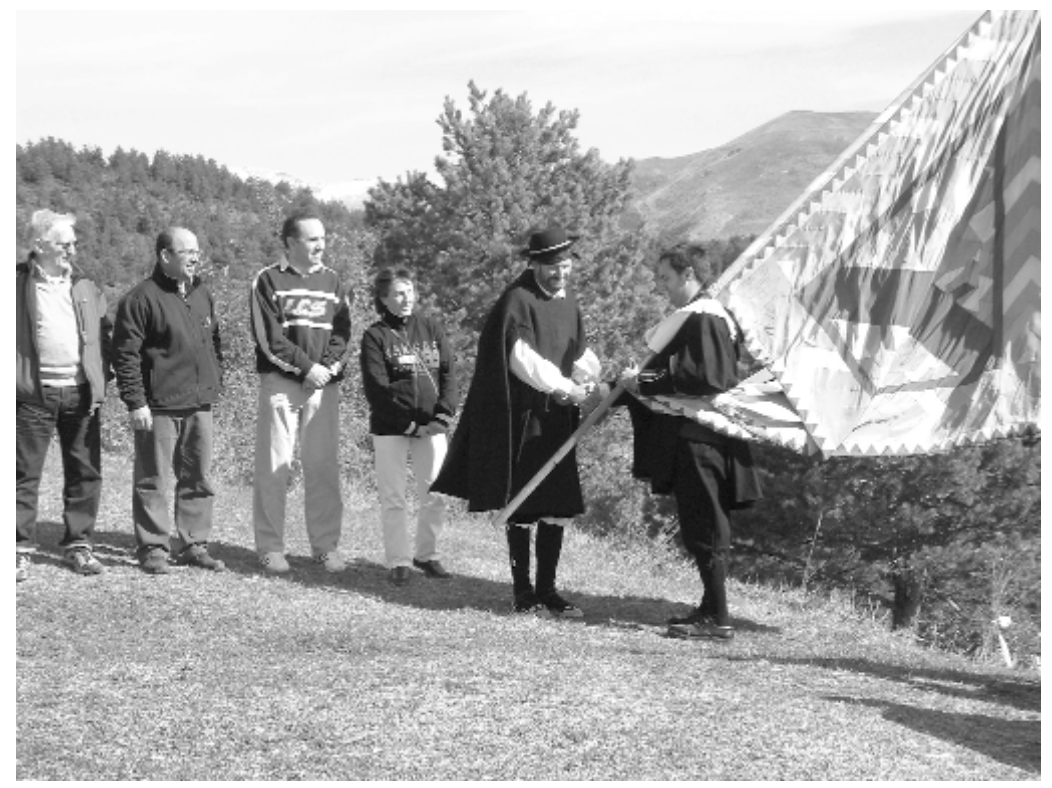

Foto 1. Encuentro de representantes de ambos valles en los llanos de Puyeta.

\footnotetext{
${ }^{7}$ Puede considerarse como una observación superficial, pero la opinión, contrastada por este autor con habitantes de ambas Comunidades Autónomas y que forma parte de otra línea de investigación, refleja que durante los primeros años de las autonomías, los pueblos limítrofes se distanciaron en el sentido de disminuir la frecuencia con que años antes se acudía a las fiestas patronales de pueblos próximos ubicados en Comunidades distintas. Las poblaciones limítrofes de ambas regiones se volcaron hacia sus respectivas Comunidades, abandonando parte de las relaciones que mantenían tradicionalmente con sus vecinos.

${ }^{8}$ Decimos sobre todo la Junta de Puyeta puesto que esta reunión conlleva el planteamiento de problemas y oportunidades mientras que en la Piedra de San Martín (Tratado de las tres vacas) el objetivo es más conmemorativo o festivo y no tanto de planificación de actuaciones.
} 
Los ritos actuales conservan o han recuperado la tradición. Las autoridades roncalesas llegan ataviadas con su bandera y traje tradicional, recibiéndolos los ansotanos con su alcalde que también viste la indumentaria ansotana. Tras la ceremonia de recepción se inicia una mesa de trabajo, exponiéndose los problemas para los que se solicita apoyo y, simultáneamente, se estudian posibles vías de colaboración que beneficien a todos. Así, entre los distintos asuntos planteados en los tres últimos años destaca:

- La reintroducción del oso en los valles. La reintroducción de osos procedentes de países del Este de Europa está generando más problemas que los osos pirenaicos autóctonos, ya desaparecidos. Los reintroducidos son de mayor tamaño, tienen "costumbres distintas" como aproximarse en exceso a las poblaciones con el peligro que esto conlleva. Los debates permiten descubrir las diferencias entre las indemnizaciones que reciben los ganaderos navarros y aragoneses, más elevadas para los ganaderos roncaleses y de menor cuantía para los ansotanos. Se planifican reivindicaciones conjuntas en este sentido.

- La mejora de las comunicaciones entre poblaciones limítrofes. Al ser carreteras de titularidad autonómica, los gobiernos regionales trabajan en el acondicionamiento y mejora de la accesibilidad hasta los núcleos de población. Pero el acondicionamiento de las carreteras desde dichos núcleos a los límites regionales o la limpieza de la nieve (básica para el acceso a las pistas de esquí de fondo) están en un segundo orden. En consecuencia, esta mejora de la accesibilidad es básica en la planificación de proyectos comunes.

- El diseño de un espacio común para el impulso turístico. El turismo se concibe como una de las principales oportunidades de desarrollo económico en la actualidad. En este sentido, los valles de Baretous, Roncal y Ansó diseñan no sólo rutas turísticas de promoción y gestión conjunta, por ejemplo la conexión de las estaciones de esquí de Arette (Baretous), Belagoa (Isaba), Linza (Ansó); la ruta de Camille (el último oso autóctono que habitó estos valles) o la ruta de las golondrinas, esta última recupera el itinerario que las mujeres de Roncal y Ansó utilizaban para desplazarse andando a trabajar a Mauleón (Béarn) en la fabricación de alpargatas. Junto a estas rutas se trabaja en la definición de un espacio educativo que ya está dando sus primeros frutos en la organización de programas culturales, sobre todo entre Roncal y Baretous bien conectados mediante vías de comunicación.

La planificación de estos proyectos requiere lazos de colaboración más complejos que si se tratase de territorios gestionados por el mismo gobierno regional, una colaboración que se sella en pactos, actas y manifiestos. Interesa resaltar uno de los párrafos del Manifiesto de Puyeta (2009), leído por el Alcalde de Ansó, Felix Ipas, que en referencia a los conflictos de otras épocas dice desde Puyeta, ansotanos y roncaleses nos sentimos comprometidos a no reconocer, nunca, otra arma que no 


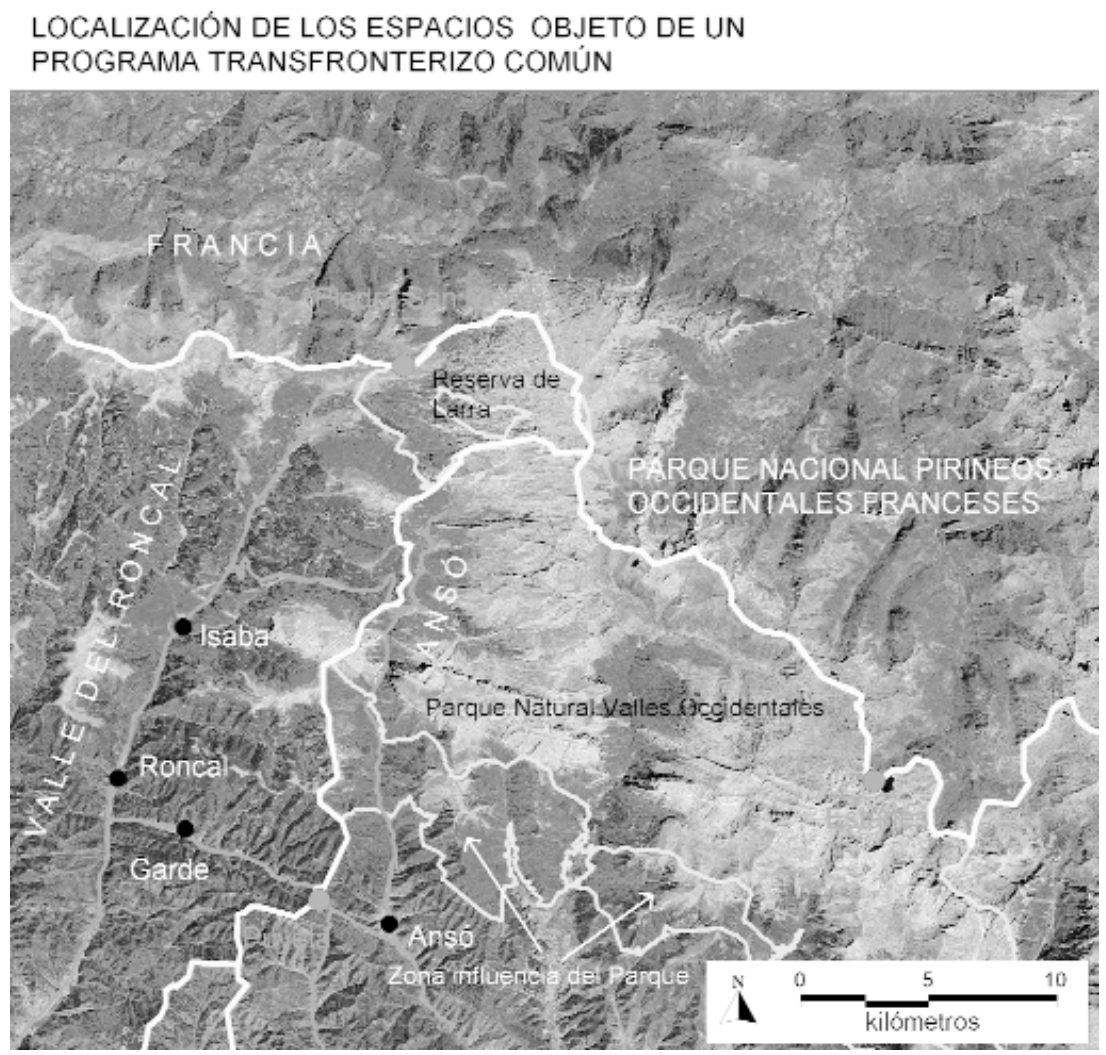

sea la palabra. El valor de ésta es sagrado en el Pirineo, con ella bemos sellado siempre nuestros pactos, con ella bemos solucionado siempre nuestros problemas. Que nunca más volvamos a sufrir las consecuencias de baber prescindido de la palabra. La recuperación de este rito supone encarar y planificar el futuro basándose en la experiencia y solidez del pasado pero en un contexto más fácil (ahora no existen "graves conflictos"), aunque también más difícil, en el sentido de que hoy estas poblaciones no cuentan con los recursos y autonomía suficiente para ser ellos mismos los planificadores del territorio, como lo hacían en el pasado.

\subsection{La biomasa como nuevo sistema de aprovechar los recursos forestales}

Entre los proyectos que se estudian conjuntamente destaca la biomasa. Este sistema de aprovechamiento de los recursos forestales constituye una alternativa a la crisis de la industria forestal. Tanto los valles navarros como aragoneses han tenido tradicionalmente en el bosque la materia prima para desarrollar su actividad industrial. 
Una actividad que llegó a ocupar mayor número de trabajadores que la propia ganadería, entre empleados en las serrerías, picadores del monte y transportistas. Durante décadas, este ritmo de extracción fue superior a la regeneración natural de los bosques, de tal forma que hacia mitades de los años noventa se vieron obligadas al cierre las serrerías de Ansó y otras del Pirineo aragonés.

Se inició así una "moratoria" en la extracción forestal del Pirineo aragonés con objeto de que se regenerase el medio forestal. Por otra parte, la calidad actual de la madera ${ }^{9}$ no permite pensar en explotaciones forestales industriales como antaño, sino en rentabilizar estos recursos obteniendo productos como pellets para calefacción u otros productos de baja calidad. Con el desarrollo de la biomasa se pretende alcanzar dos objetivos, uno la creación de puestos de trabajo arraigados en el territorio ${ }^{10}$ $y$, otro, conseguir mejoras ambientales en el sentido de que buena parte de los recursos que utiliza la biomasa proceden de la limpieza del monte y este debe mantenerse limpio para reducir los riesgos de incendios.

La obtención de energía a partir de la biomasa puede ser una solución de futuro. No obstante, se debe aprender de errores del pasado para no hipotecar el futuro. Nos estamos refiriendo a que las necesidades de materia prima para que esta actividad sea rentable rondan las $10.000 \mathrm{~T} / \mathrm{año}$, un volumen semejante a la masa forestal que se extraía en el término municipal de Ansó en los momentos de máxima explotación y que demostraron su insostenibilidad, lo cual exige obtener materia prima fuera del término municipal; es decir, una gestión conjunta o supramunicipal de este recurso. Como punto fuerte es la generación de mano de obra en el monte que puede ser incluso superior a la existente antaño, puesto que es más costoso obtener 1 tonelada de residuos que de madera. ${ }^{11}$ Un proyecto que nació con proyección "transregional" precisamente para obtener materia prima suficiente, si bien la evolución de los acontecimientos ha desembocado en impulsar una central de biomasa en cada valle. La de Ansó ya comenzó a funcionar en octubre de 2011.

A pesar de su interés la mayor preocupación es garantizar unos sistemas de gestión y ordenación bien planificados. En el proyecto de explotación se dice que no se

\footnotetext{
${ }_{9}$ Durante décadas se explotaron especies de gran calidad como el haya y el abeto que son las que más se han reducido, permaneciendo en buen estado solo aquellas masas que se ubican en zonas de difícil accesibilidad. Hoy sólo el pino reúne condiciones para su explotación pero tampoco cuenta con calidad para competir en el mercado de la madera, de ahí que la biomasa constituya la principal vía para la rentabilidad económica-industrial del medio forestal.

${ }^{10} \mathrm{La}$ industria se considera (no sólo desde estos valles) como el sector con mayor capacidad para arraigar a la población en el territorio, sobre todo teniendo en cuenta que la ganadería es muy difícil que genere mayor ocupación de la actual. Por su parte, el turismo en pocos casos es la actividad principal, sino más bien, y sobre todo en la actualidad, complementaria a otros sectores.

${ }^{11}$ También como punto fuerte destacamos el apoyo Institucional a esta fuente de energía. En este sentido el Gobierno de Aragón participa con distintos países de la cuenca del Mediterráneo en un proyecto europeo para optimizar el suministro energético a partir de la biomasa.
} 


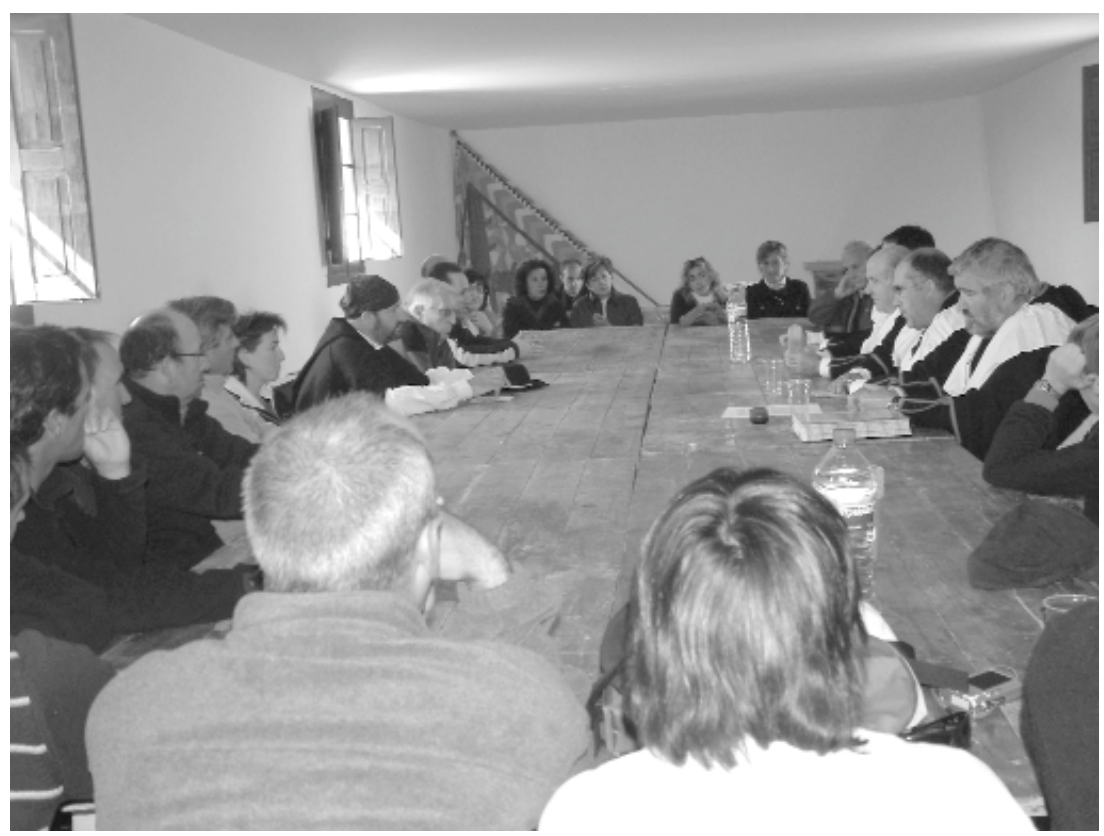

Foto 2. Sesión de debate en la Junta de Puyeta.

utilizarán árboles maduros, sino aquellos con un diámetro inferior a 40 ó $50 \mathrm{~cm}$, pero no debemos olvidar que estos árboles jóvenes son los que deben regenerar el bosque, luego hay que hacer una ordenación muy precisa. Habrá que pensar en la plantación de cultivos energéticos, especies forestales de crecimiento rápido, creación de viveros para los plantones de cultivos energéticos... Es decir, se abren nuevas expectativas pero que requieren minuciosos estudios, planes y gestión supramunicipal, pues, en principio, parece difícil un total abastecimiento de materia prima en cada valle.

\subsection{Reducir el impacto de los residuos ganaderos en el medio ambiente}

La ganadería ha descendido en el conjunto de estos valles, pero la generación de residuos (estiércol) genera mayores problemas que antaño. Esto es así porque, en el pasado, la ganadería funcionaba mediante sistemas de explotación extensivos, ligados a la tierra, y los residuos se incorporaban al suelo como materia orgánica en un proceso natural, rico para la ecología y sin costes para el ganadero. En estos momentos, los sistemas de explotación requieren una mayor permanencia del ganado en los establos con lo cual el estiércol se acumula y, más que suponer un beneficio al monte 
(abonado) genera un problema pues no existen en estos valles terrenos agrícolas suficientes para asumirlos.

La solución que se estudia es la construcción de una planta de biogás. Un proyecto que nació unido, o paralelo, al desarrollo de la biomasa, pero de mayor complejidad económica y medioambiental por lo que todavía se halla en fase de estudio. Una planta (pensada para Ansó) que necesitaría el aporte de residuos desde otros valles, tanto Roncal como Echo. En este proyecto más que la obtención de beneficios económicos (como es la biomasa) se trata de solucionar un problema medioambiental que afecta a los ganaderos, y administraciones públicas, de los distintos valles.

\subsection{La apuesta por la terciarización}

El turismo emerge en estos valles como una buena alternativa al sistema económico tradicional, no obstante se detectan muchas reticencias por la fuerte estacionalidad. Los recursos para planificar un turismo de calidad existen: parque natural de los valles occidentales (en Aragón), reserva natural de Larra (Navarra), parque nacional de los Pirineos Occidentales (Francia), pistas de esquí de fondo en los valles españoles y alpino en Arette, el día de la exaltación del traje tradicional ansotano, el descenso almadiero por el Esca, la propia celebración del Tributo de las Tres Vacas... son los ejemplos más conocidos, pero no los únicos, para generar un producto turístico de los valles occidentales.

Y en esta dirección van los esfuerzos de las Administraciones locales. En Ansó, aprovechando la existencia del parque natural (y una vez asumido éste, que no fue fácil) se piensa en avanzar más y crear aulas pedagógicas a modo de escuelas de verano $^{12}$ de la naturaleza. En Roncal se va más lejos y se está diseñando una Universidad virtual (un colaboración con departamentos de las Universidades de Navarra y del País Vasco). Ambos proyectos se complementarían con la vertiente francesa y, entre todos, se plantea un ambicioso proyecto Interreg que, de llegar a "buen puerto", se puede convertir en la mayor plataforma de cooperación transfronteriza que ha existido en estos valles en las últimas décadas.

\section{A modo de conclusión}

Estamos ante un espacio donde se desarrolló un complejo e interesante sistema de relaciones transfronterizas. Relaciones que llegaron a desaparecer totalmente pero

\footnotetext{
${ }^{12} \mathrm{O}$ de otoño con objeto de desestacionalizar el turismo y atraer actividad fuera del verano. En la época estival no se puede incrementar más la actividad turística pero las temporadas de inactividad son muy grandes, lo que obliga a considerar el turismo como una actividad complementaria, al menos en el momento actual, sino se logra una eficaz desestacionalización.
} 
que en estos momentos se abren nuevas expectativas para volver a integrar los valles en proyectos comunes. Los instrumentos para canalizar dichos proyectos existen (Interreg, Acuerdos de Cooperación entre los gobiernos regionales...), diferentes normativas de ámbito estatal (ley 45/2007 de Desarrollo Rural Sostenible en el caso del Estado español) unos y otros permiten avanzar en este proceso. Un proceso que podemos denominar como reencuentro y cuyo punto fuerte es que se hace contando con buenos recursos y con base en experiencias históricas que funcionaron bien. Evidentemente, nace en situaciones que tienen poco que ver con el pasado, pero éste, desde el punto de vista cultural, conllevan un fuerte valor simbólico en la eliminación de fronteras y enlace de pueblos y no deja de ser un buen punto de partida.

\section{Bibliografía}

Balcells, E. (1976). El Pirineo: contraste de paisajes; enlace pueblos. Conferencia pronunciada en la Comisión Internacional de los Pirineos. Madrid.

Bielza de Ory y Dalla-Rossa, G. (1988). Las relaciones transpirenaicas. Colección "cosas nuestras" Instituto de Estudios Altoaragoneses. Huesca.

Cavailles, H. (1910). Une fédération pyrénéene sous l'Ancien Regime. Les Traités de Lis et Passeries. Revue Histórique, sep-déc., pp $1-34$ y $241-276$.
Caussimont, G. (1981). Etude compare des commnunautes des valles de Hecho, Ansó, Roncal, Haut-Ossau, Aspe, Baretous. (L'exemple de una crise d'une société montagnarde, de su estructure aux mentalites), Université de Pau. Pau.

Fairén-Guillén, V. (1956). Facerias Internacionales Pirenaicas. Instituto de Estudios Políticos. Madrid.

Gorría Ipas, A.J. (1995). El Pirineo como espacio frontera. Diputación General de Aragón. Zaragoza. 